\title{
Accuracy Improvement in CCT Estimation of Power Systems by iRprop-RAN Hybrid Neural Network
}

\author{
Teruhisa Kumano, Shinjiro Netsu \\ Department of Electronics and Bioinformatics, Meiji University, Kawasaki, Japan \\ Email: kumano@isc.meiji.ac.jp \\ Received March, 2013
}

\begin{abstract}
This paper proposes a new Initial CCT (Critical Clearing Time) estimation method using a hybrid neural network composed of iRprop (Improving the Resilient back PROPation Algorithm) and RAN (Resource Allocation Network). In transient stability study, CCT evaluation is very important but time consuming due to the fact it needs many iteration of time domain simulations gradually increasing the fault clearing time. The key to reduce the required computing time in this process is to find accurate initial estimation of CCT by a certain handy method before going to the iterative stage. As one of the strongest candidates of this handy method is the utilization of the pattern recognition ability of neural networks, which enable us to jump to a close estimation of the real CCT without any heavy computing burden. This paper proposes a new hybrid neural network which is a combination of the well-known iRprop and RAN. In the proposed method, the outputs of the hidden units of RAN are modified by multiplying the contribution factors calculated by an additional iRprop network. Numerical studies are done using two different test systems for the purpose of confirming the validity of the proposal. The result of the proposed method is the best. Properly evaluating the contribution of each input to the hidden units, the estimation error obtained by the proposed method is improved further than the original RAN based estimation.
\end{abstract}

Keywords: Critical Clearing Time; Estimation; Power System; iRprop; Resource Allocation Network

\section{Introduction}

In large power systems operation, stability check in the contingency analysis is always important. In particular, in these two years, we, Japanese electric power engineers, cannot depend upon the strong power supply from the nuclear power plants and the basic power flow pattern is different from the original schedule. It implies that every daily grid operation needs more careful study, in which stability check should be done. The long and narrow power corridor is, in a sense, inevitable due to the geographical restriction of our country, which makes the stability the main restricting factor for the long and heavy power transmission.

One of the biggest problems in the transient stability constrained contingency analysis is the long computing time required. Transient stability study itself is a typical time consuming calculation. Here, we need to iterate dynamic simulation many times gradually increasing power flow or fault clearing time to reach their critical value. Comparing these two, the critical clearing time (CCT) is easy to calculate and is often used. For the fast evaluation of CCT, we need accurate initial guess of the clearing time. If we can start from close guess, the required time to get the true CCT can be shorter. Since the power flow pattern in the whole system gives a substantial effects on the resultant CCT, it can be expected that we can accurately estimate CCT if the important variables such as the specified values of the active and reactive power at each node is given.

Many methods based on so called artificial neural network (ANN) techniques have been studied for this initial guess. Ikenono et al proposed to use BP (back propagation) based ANN [1]. Bettiol et al proposed to use RBF (Radial Basis Function) network for this purpose [2]. The authors ourselves studied this problem and proposed to use support vector machine [3] and relevance vector machine [4]. Once properly trained, ANN can recognize the given input pattern and make classification or give a regression in a short computing time. Because of its nonlinear knowledge representation ability, it has been a strong candidate for this initial estimator. However, even after the above mentioned research efforts, it still remains as a research theme and not a real field application.

In this paper, a new ANN method is proposed for the above stated purpose. In this proposal so called RAN (Resource Allocation Network) is coupled with iRprop (Improving the Rprop Learning Algorithm, in which 
Rprop stands for Resilient backPROPagation). RAN is one type of RBF network, but it has a strong adaptability given by the hidden layer adjustment. RAN and its modifications are applied to various fields and makes a considerable contribution. [5-7] The details of iRprop and RAN will be described in the next chapter.

This paper is constructed as follows. Chapter 2 explains the proposal in this paper together with the basics related to iRprop and RAN. Chapter 3 describes our transient stability problem, followed by numerical examples written in Chapter 4. In Chapter 5 concluding remarks are stated.

\section{Related Networks and the Proposal}

\section{1. iRprop}

The proposed method in this paper is a hybrid of iRprop and RAN. The former (more strictly, iRprop ${ }^{+}$) is an improved version of BP (Back Propagation) ANN. While BP shifts the weight of each connection by the gradient and needs the partial derivative calculated, iRprop only watches the sign of the partial derivative. Its light computing cost enables us to apply this method to many fields.

The mathematical description of its learning algorithm is as follows [8]. The weight of each connection is $w_{i j}$ and it is shifted by the following algorithm depending upon the sign of $\frac{\partial \mathrm{E}^{(\mathrm{t}-1)}}{\partial \mathrm{w}_{\mathrm{ij}}} \cdot \frac{\partial E^{(t)}}{\partial w_{i j}}$, where $E$ stands for the sum of the output error. In the following equations, coefficient $\eta^{+}$and $\eta^{-}$should satisfy $0<\eta^{-}<1<\eta^{+}$. $\Delta_{\max }$ and $\Delta_{\min }$ imply the upper and lower limits of $\Delta_{i j}^{(t)}$

$$
\begin{gathered}
\text { i) In case } \frac{\partial \mathrm{E}^{(t-1)}}{\partial \mathrm{w}_{\mathrm{ij}}} \cdot \frac{\partial E^{(t)}}{\partial w_{i j}}>0 \\
\Delta_{i j}^{(t)}=\min \left(\Delta_{i j}^{(t-1)} \cdot \eta^{+}, \Delta_{\max }\right) \\
\Delta w_{i, j}^{(t)}=-\operatorname{sign}\left(\frac{\partial E^{(t)}}{\partial w_{i j}}\right) \cdot \Delta_{i j}^{(t)} \\
w_{i j}^{(t+1)}=w_{i j}^{(t)}+\Delta w_{i j}^{(t)} \\
\text { ii) in case } \frac{\partial \mathrm{E}^{(t-1)}}{\partial \mathrm{w}_{\mathrm{ij}}} \cdot \frac{\partial E^{(t)}}{\partial w_{i j}}<0 \\
\Delta_{i j}^{(t)}=\max \left(\Delta_{i j}^{(t-1)} \cdot \eta^{-}, \Delta_{\min }\right) \\
\text { if } E^{(t)}>E^{(t-1)} \text { then } w_{i j}^{(t+1)}=w_{i j}^{(t)}-\Delta w_{i j}^{(t-1)} \\
\text { otherwise } w_{i j}^{(t+1)}=w_{i j}^{(t)} \\
\frac{\partial E^{(t)}}{\partial w_{i j}}=0 \\
\text { iii) in case } \frac{\partial \mathrm{E}^{(t-1)}}{\partial \mathrm{w}_{\mathrm{ij}}} \cdot \frac{\partial E^{(t)}}{\partial w_{i j}}=0
\end{gathered}
$$

$$
\begin{aligned}
& \Delta w_{i, j}^{(t)}=-\operatorname{sign}\left(\frac{\partial E^{(t)}}{\partial w_{i j}}\right) \cdot \Delta_{i j}^{(t)} \\
& w_{i j}^{(t+1)}=w_{i j}^{(t)}+\Delta w_{i j}^{(t)}
\end{aligned}
$$

\subsection{RAN}

On the other hand, RAN is composed of 1) a network, 2) strategy for allocating new units, and 3) a learning rule for refining the network. [9] It is based on the concept of RBF (Radial Basis Function) machine. [2] Let $I$ be the input. Using $c$ and $\sigma^{2}$, the center and the variance of the RBF, $y$, the output of the hidden unit, is computed by

$$
y_{i}=\exp \left(-\left\|I_{p}-c_{j}\right\|^{2} / \sigma_{j}^{2}\right)
$$

where $j, i$, and $p$ stand for the suffix for the number of the hidden units, those of the input units, and sample id respectively. The network output $z$ is calculated, using the weight $h$ and the output bias $\gamma$, as follows.

$$
z_{k}=\sum_{i} h_{k} y_{i}+\gamma_{k}
$$

RAN also learns so as to minimize the sum of the squared output error $E$. When the newly obtained input is not found in the experience so far (far from the used data sample obtained in the history), another hidden unit is added. This algorithm is formulated as follows.

$$
\begin{aligned}
& \text { if } E>\varepsilon \text { and }|| x \text { - } C_{\text {nearest }}||>\delta \text { then } \\
& \begin{array}{c}
j=j+1 \\
c_{j i}=x_{p i} \\
\sigma_{j}^{2}=k\left\|x-c_{\text {nearest }}\right\| \\
h_{j i}=T_{p k}-z_{k}
\end{array}
\end{aligned}
$$

where $T_{p k}$ is the teacher signal.

\subsection{The Proposed Method}

RAN modifies its structure by adding a new hidden unit only in the case where the output error and the difference between the input data and the nearest center of the existing radial basis functions are big enough simultaneously. However, in our specific problem, the input data contain various quantity such as voltage, power, and fault location etc.. Some input might have strong effects on the inputs to the hidden layer, but others not. In the proposed method, this difference in the degree of the effects on the hidden layer is assessed by an additional iRprop network which connects every input variable with every hidden unit.

Learning is done simultaneously in this additional iRprop network and the obtained weight of each connection is multiplied to the difference between each input and the nearest center, the sum of which is used to calculate the output of the hidden units. Using $y, I, c$, and $\sigma$ of the same meaning as in the previous section, the output of 
the hidden unit $y$ is written as

$$
y_{i}=\exp \left(-\frac{\left\|I_{p}-c_{j}\right\|^{2} e f_{i}^{2}}{\sigma_{j}^{2}}\right)
$$

where the new coefficient ef implies the degree of effects and is calculated by the following formula;

$$
e f_{i}=\sum_{j} w_{i j},
$$

which is the sum of the weights in the above stated additional iRprop network. The proposed network takes the form shown in Figure 1. The additional part on the top (boxed in the broken rectangle) is iRprop, the sum of the connection weight of which is ef and is multiplied to the distance between input $I_{k}$ and the nearest center as shown in Equation (3). Figure 2 shows the type of input/output data used in the proposed method.

\section{Studied Problem}

\subsection{Data Preparation Procedure}

In our particular problem, so called CCT (Critical Clearing Time) is the target of the estimation. CCT is the

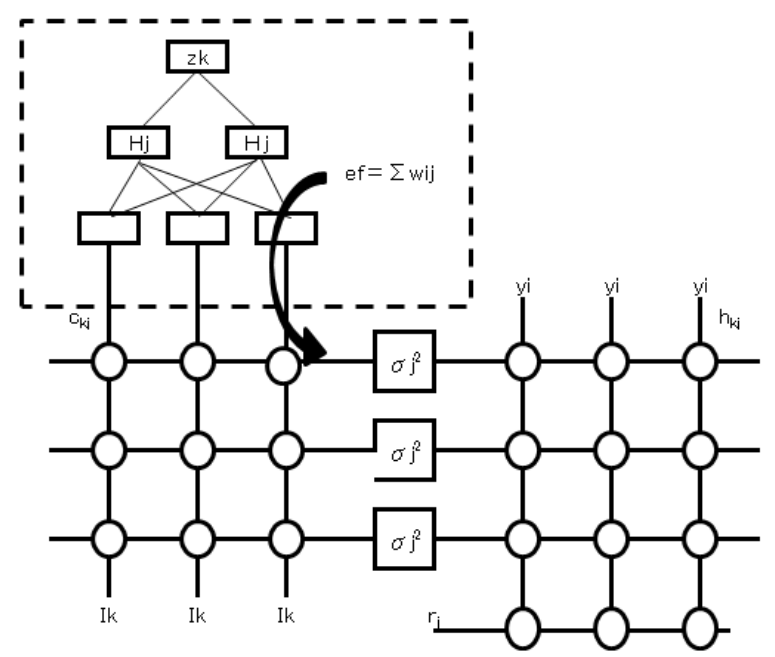

Figure 1. The structure of the proposed network.

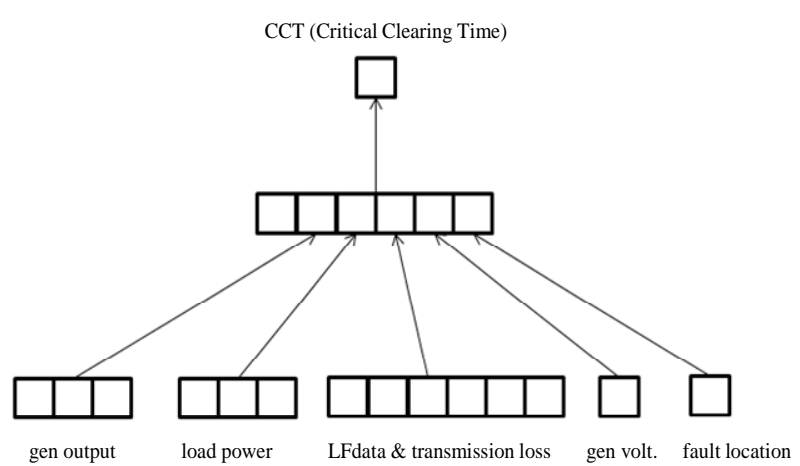

Figure 2. Inputs and output of the proposed network. maximum fault duration which does not cause instability. In order to obtain the teacher signal fed to the proposed method, the true CCT, dynamic simulation is run many times. It is true that this is only a result of simulation analysis, but is treated as the correct CCT.

Using CPAT (CRIEPI's Power system Analysis Tool) [10], a de facto standard stability analysis program in many Japanese power utilities, the power system transient behavior is computed under various conditions such as initial load flow, fault location etc., where the fault clearing time is gradually increased. The assumed longest clearing time which causes no transient stability problem such as out of step within the predetermined time range (described later in Tables $\mathbf{1}$ and 2) is considered as CCT.

Table 1. Simulation Condition in 9 Node System.

\begin{tabular}{ll}
\hline item & setting \\
\hline gen. output & $\begin{array}{l}\text { increased at the constant rate to keep up with } \\
\text { the total demand }\end{array}$ \\
load demand & $\begin{array}{l}\text { 80, 90, 100, 110, 120\% } \\
\text { gen and load power, branch power flow, nodal } \\
\text { input data }\end{array}$ \\
voltage, branch loss, fault location \\
output & CCT \\
fault locations & sending end of all branches \\
fault type & 3LG (three lines grounded) \\
criterion of SO & internal bus angle > 180 deg \\
etc & simulation quits after t=0.5sec \\
\hline
\end{tabular}

\begin{tabular}{|c|c|}
\hline item & setting \\
\hline gen. output & $\begin{array}{l}\text { output from a gen in Area } A \text { is increased by } \\
0.1 \mathrm{pu} \text { step while the output from all gens in } \\
\text { Area B are reduced at the same rate to keep the } \\
\text { total demand unchanged }\end{array}$ \\
\hline load demand & $100 \%$ unchanged \\
\hline input data & $\begin{array}{l}\text { gen power, branch power flow, nodal voltage, } \\
\text { fault location }\end{array}$ \\
\hline output & CCT \\
\hline fault locations & $\begin{array}{l}\text { at } \mathrm{HV} \text { bus of the step-up transformer of gen- } \\
\text { erators } 8,9,10\end{array}$ \\
\hline fault type & 3LG (three lines grounded) \\
\hline criterion of SO & internal bus angle $>180$ deg \\
\hline etc & simulation quits after $\mathrm{t}=4.0 \mathrm{sec}$ \\
\hline
\end{tabular}

Table 2. Simulation Condition in 47 Node System.

\subsection{Study Power Systems}

Two model systems shown in Figures $\mathbf{3}$ and $\mathbf{4}$ are studied. The details such as machine constants used in the 9 bus model can be found in [11]. In the study of the 47 
node system, base loading is set as its standard, or $100 \%$ loading condition. The analysis condition is summarized in Tables 1-3.

\subsection{Transient Stability Analysis}

The transient stability analysis is done, which is required to get the input and the teacher signal fed to our ANN, as explained below. The simulation condition used for the nine bus model is shown in Table 1. On the other hand, Table 2 shows that used in 47 node model. In Table 2, two areas are refereed in the description of the procedure how the generator outputs are changed. Area A means the northern part of the 47 node system, which includes the three generators G8 - G10. The remaining region is Area B. Tables $\mathbf{3}$ and $\mathbf{4}$ show the generation/demand settings for each case.

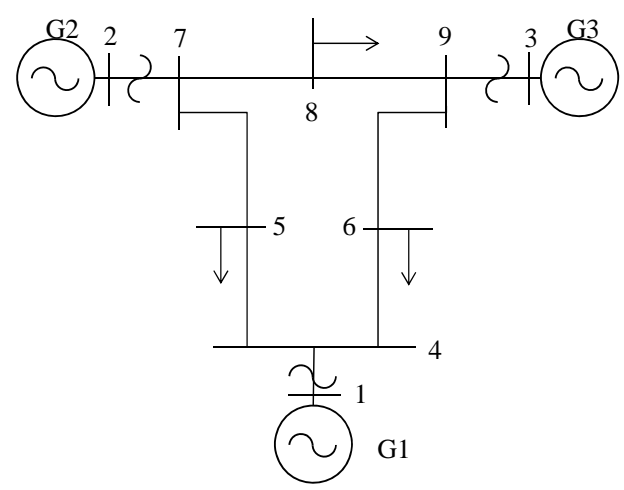

Figure 3. Anderson and Fouad 9 bus system.

\section{Numerical Study}

For the purpose of confirmation of the validity of the proposed method, it is compared with the two existing networks, BP (the classical Back Propagation based network) and iRprop. The parameters are determined by try and error. They are summarized in Tables 5-6.

Table 3. Generator Condition in 47 Node System.

\begin{tabular}{|c|c|c|c|}
\hline gen No & gen type & voltage & capacity \\
\hline 1 & LNG & $1.01 \mathrm{pu}$ & 8240MVA \\
\hline 2 & nuclear & $1.00 \mathrm{pu}$ & 12940MVA \\
\hline 3 & pumped storage & $1.02 \mathrm{pu}$ & 7060MVA \\
\hline 4 & coal & $1.01 \mathrm{pu}$ & 12940MVA \\
\hline 5 & pumped storage & $1.02 \mathrm{pu}$ & 7060MVA \\
\hline 6 & coal & $1.02 \mathrm{pu}$ & 12940MVA \\
\hline 7 & nuclear & $1.00 \mathrm{pu}$ & 12940MVA \\
\hline 8 & oil & $1.00 \mathrm{pu}$ & 8240MVA \\
\hline 9 & oil & $1.00 \mathrm{pu}$ & 8240MVA \\
\hline 10 & oil & $1.02 \mathrm{pu}$ & 5880MVA \\
\hline
\end{tabular}

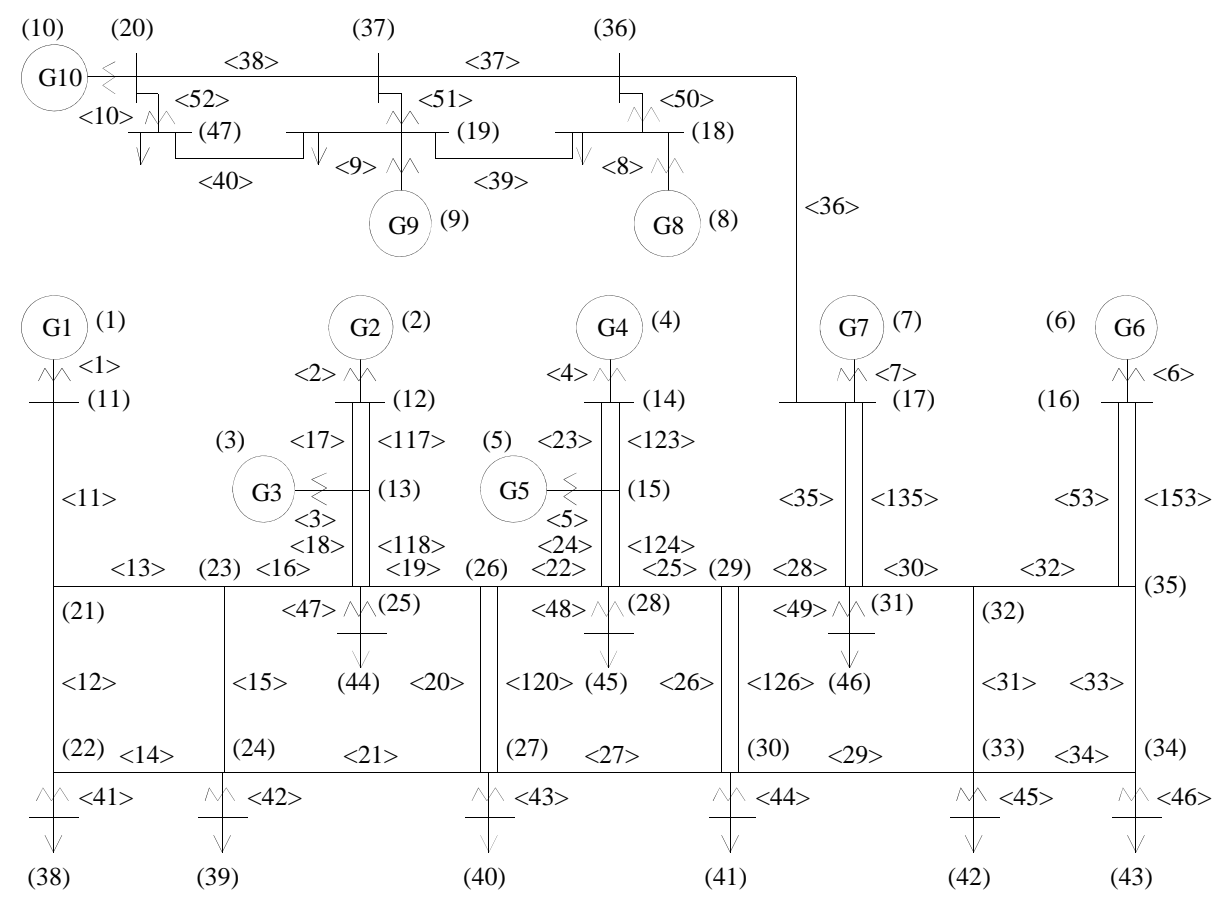

Figure 4. IEEJ standard 47 bus model system EAST-10. 
Table 4. Load Condition in 47 Node System.

\begin{tabular}{ccc}
\hline Node No & active power & reactive power \\
\hline 38 & $3.5 \mathrm{pu}$ & $0.986 \mathrm{pu}$ \\
39 & $7.0 \mathrm{pu}$ & $1.972 \mathrm{pu}$ \\
40 & $7.0 \mathrm{pu}$ & $1.972 \mathrm{pu}$ \\
41 & $7.0 \mathrm{pu}$ & $1.972 \mathrm{pu}$ \\
42 & $7.0 \mathrm{pu}$ & $1.972 \mathrm{pu}$ \\
43 & $3.5 \mathrm{pu}$ & $0.986 \mathrm{pu}$ \\
44 & $3.5 \mathrm{pu}$ & $0.1 \mathrm{pu}$ \\
45 & $3.5 \mathrm{pu}$ & $0.1 \mathrm{pu}$ \\
46 & $3.5 \mathrm{pu}$ & $0.1 \mathrm{pu}$ \\
18 & $3.85 \mathrm{pu}$ & $1.205 \mathrm{pu}$ \\
19 & $3.85 \mathrm{pu}$ & $1.205 \mathrm{pu}$ \\
47 & $2.8 \mathrm{pu}$ & $0.806 \mathrm{pu}$ \\
\hline
\end{tabular}

Table 5. Settings in BP.

\begin{tabular}{ccccc}
\hline $\begin{array}{c}\text { Hidden } \\
\text { units }\end{array}$ & $\begin{array}{c}\text { Learning } \\
\text { rate }\end{array}$ & treshold & $\begin{array}{c}\text { Initial } \\
\text { setting }\end{array}$ & $\begin{array}{c}\text { max learning } \\
\text { iteration }\end{array}$ \\
\hline 132 & 0.2 & 0.0 & $\begin{array}{c}\text { randomly set } \\
\text { between -1.0 - 1.0 }\end{array}$ & 6000 \\
\hline
\end{tabular}

Table 6. Settings in iRprop.

\begin{tabular}{ccccc}
\hline $\begin{array}{c}\text { hidden } \\
\text { units }\end{array}$ & $\begin{array}{c}\text { initial } \\
\text { update }\end{array}$ & $\begin{array}{c}\text { max/min } \\
\text { update }\end{array}$ & initial setting & $\begin{array}{c}\text { max learning } \\
\text { iteration }\end{array}$ \\
\hline 30 & 0.5 & $50,10^{-6}$ & $\begin{array}{c}\text { randomly set } \\
\text { between -1.0 - 1.0 }\end{array}$ & 10000 \\
\hline
\end{tabular}

The obtained results are summarized in Table 7 for the 9 node system, and in Table 8 for the 47 node system respectively. Both in these two systems, the proposed method, and the hybrid iRprop/RAN network gives the best results. In our preliminary study, the existing RAN network has also been applied to these two networks. The results are shown in Table 9. This table shows the fact that RAN itself has certain relative advantage over the other existing networks, BP and iRprop. As far as the authors know, the application of RAN to this problem is the first attempt, but in this paper, RAN is further improved to the hybrid network, and this proposed method gives the best result.

The main reason why the proposed method gives the better results compared to RAN is, as the authors estimate, the contribution of the additional iRprop network. Because iRprop itself has a certain strength in this CCT estimation problem, it appropriately extracts the input feature and enables us to judge the necessity of adding a new hidden unit. The maximum error in the 9 node system becomes worse than in case of the conventional RAN but compared to the improvement from BP and iRprop, this deterioration is very small.
Table 7. Resultant Error in the 9 node system.

\begin{tabular}{cccc}
\hline & BP & iRprop & proposed net \\
\hline average & $0.0674 \mathrm{sec}$ & $0.0396 \mathrm{sec}$ & $0.0191 \mathrm{sec}$ \\
$\max$ & $0.2488 \mathrm{sec}$ & $0.2646 \mathrm{sec}$ & $0.1131 \mathrm{sec}$ \\
\hline
\end{tabular}

Table 8. Resultant Error in the 47 node system.

\begin{tabular}{cccc}
\hline & BP & iRprop & proposed net \\
\hline average & $0.00249 \mathrm{sec}$ & $0.00263 \mathrm{sec}$ & $0.00208 \mathrm{sec}$ \\
$\max$ & $0.00884 \mathrm{sec}$ & $0.01041 \mathrm{sec}$ & $0.00691 \mathrm{sec}$ \\
\hline
\end{tabular}

Table 9. Error in Case of Conventional RAN.

\begin{tabular}{ccc}
\hline & 9 node system & 47 node system \\
\hline average & $0.03003 \mathrm{sec}$ & $0.00229 \mathrm{sec}$ \\
$\max$ & $0.09311 \mathrm{sec}$ & $0.00855 \mathrm{sec}$ \\
\hline
\end{tabular}

Since the maximum error is reduced to be around $70 \%$ of that by the existing method, the required computation time is also expected to be around $70 \%$.

Summarizing all these points, it can be said that the proposed method gives significant improvement on the initial CCT guess, and it implies that the total computing time required to get the true CCT can be shorten considerably.

\section{Conclusions}

In this paper, a new ANN based CCT estimation method is proposed to realize a faster power system transient study. The proposed ANN is a hybrid of iRprop and RAN, in which additional iRprop network gives helpful information concerning the new hidden unit generation in the conventional RAN.

Numerical study is done to confirm the validity of the proposed method in the CCT estimation problem in the power system stability analysis, where several existing methods are also tested and compared. The existing RAN is used for this purpose, which is, as far as the authors know, also the first attempt so far, and gives a better result compared to other existing methods such as BP and iRprop. Furthermore, the proposed method gives an even better result in the sense of the maximum and averaged estimation error.

In the present paper, initial load flow pattern and, needless to say, the fault location are varied and the proposed method is shown to be able to give a fairly good result in a wide range of the condition. However, no topological change is considered in the model system. Since the addition of the iRprop network might have given a strong ability of adapt and adjust to new operating condition in the proposed method, it can be expected 
that our method might be well applied in a bigger condition change such as topological. Due to the new grid participants such as distributed generators, the grid configuration might be more frequently changed from now, which might affects the power transmission capability of the trunk system. It implies that the importance of the proposed method will become bigger and bigger in near future.

\section{REFERENCES}

[1] K. Ikenono and S. Iwamoto, "Generalization of Transient Stability Solution using Neural Network Theory," Trans IEEJ, Vol. 111, No. 7, 1991, pp. 723-728.

[2] A. L. Bettiol, A. Souza, J. L. Todesco and J. R. Tesch, Jr, "Estimation of Critical Clearing Times Using Neural Networks,” 2003 IEEE Bologna Power Tech Conference Proceedings. doi:10.1109/PTC.2003.1304446

[3] H. Takahashi and T. Kumano, “Available Transfer Capability Screening Considering Transient Stability by Support Vector Machine,” 2008 IEEE PES General Meeting, Pittsburgh. doi:10.1109/PES.2008.4596499v

[4] A. Wada and T. Kumano, "Fast Estimation of Transient Stability Cconstrained ATC by Relevance Vector Machine," IEEE 2nd International Power and Energy Conference, 2008, doi:10.1109/PECON.2008.4762451v

[5] B. S. Mahanand, S. Suresh, N. Sundararajan, M. A. Kumar, "Alzheimer's Disease Detection Using a
Self-adaptive Resource Allocation Network classifier," The 2011 International Conference on Neural Networks, pp. 1930-1934.doi:10.1109/IJCNN.2011.6033460

[6] S. Shanthi and V. M. A. Bhaskaran, "Computer Aided Detection and Classification of Mammogram Using Self-adaptive Resource Allocation Network Classifier," 2012 International Conference on Pattern Recognition, Informatics and Medical Engineering (PRIME), pp. 284-289. doi:10.1109/ICPRIME.2012.6208359

[7] H. C. Lou and W. Z. Dai, "A Novel Non-linear Model Predictive Controller Based on Minimal Resource Allocation Network and Its Application in CSTR PH Process," 7th World Congress on Intelligent Control and Automation, 2008. WCICA 2008, pp. 5672-5676. doi:10.1109/WCICA.2008.4593855v

[8] C. Igel and M. Husken, "Empirical Evaluation of the Improved Rprop Learning Algorithms,” Neurocomputing, Vol. 50, 2003, pp. 105-123. doi:10.1016/S0925-2312(01)00700-7

[9] J. Platt, “A Resource-Allocating Network for Function Interpolation,” Neural Computation, Vol. 3, No. 2, 1991, pp. 213-225.doi:10.1162/neco.1991.3.2.213v

[10] Y. Kitauchi, "Scheme of Power System Stability Enhancement using Margin to Apparatus Limitation (Part II) - Verification of Power System Stability Improvement using Highly Voltage Control on IEEJ WEST 30-machine System Model-", CRIEPI REPORT R04010, 2005.

[11] P. M. Anderson and A. A. Fouad, Power System Control and Stability, Iowa State University Press, 1977. 